\section{A REMARKABLE CASE OF A JUVENILE EARTH-EATER.}

By CLEMENT DUKES, M.D., B.S., M.R.C.P. LoND., PHYSICIAN TO RUGBY SCHOOL, AND TO THE HOSPITAL OF ST. CROSS, RUGBY.

THE following case seems to me to be of sufficient interest for publication.

P, B-, aged five years, residing quite in the country, was brought to the Rugby Mospital on December 18th, 1883, by her mother, who carried with her in a bottle a characteristic specimen of the common round worm, ascaris lumbricoides. The mother gave the following history: Two years ago the child did not seem well, and after a day or two she became sick, and threw up a large round worm. The mother then gave her a dose of castor oil, and she passed by the bowel three more. A week after this the mother noticed that the child ate the soil in the garden, or that by the side of the road. To stop this vicious habit the child was kept indoors and scolded and whipped, but without effect ; for whenever the mother was engaged in her household duties the child would immediately run out and eat the dirt. She always said, when reprimanded, that she ate the earth to relieve the gnawing pain in her stomach. So urgent was the demand for this soil-eating that the child would, if she could not get earth, eat sand, would pick the mortar out of the walls between the bricks, and she would even store a supply in her pocket to eat at her leisure, when she could not get out to obtain it. She has continued to eat the soil, in spite of continual punishments, until the time of her entrance into the hospital on February 12th, 1884, when she ceased, as far as could be ascertained, to eat the soil. The mother states that she would put the earth in her mouth, suck it, and then swallow it. She further states that during the two years of eartheating she has brought up or passed by the bowel about a handred large round worms. From the number I have seen myself, and from the respectability of the mother, I fully believe this statement. While in hospital she passed many, but under santonin they failed to come away; and after being without any for a month, and having become fat and strong, I discharged her on April 10th, 1884, as I thought cured. But on May 2nd her mother brought her back, she having passed another worm, but she had not again taken to eating earth. During the child's stay in hospital her perverted taste had apparently been cured. I readmitted her, when she soon passed a worm, and vomited another; but under two-grain doses of santonin three times a day, which she took for several weeks without physiological effects, she gradually ceased passing any more, and regained her health, strength, and good looks.

Cobbold says, round worms are rare in British practice, and usually only one occurs: this is my experience. But he also records that Mr. de Morgan had a patient at the Middlesex Hospital from whom thirty-seven were expelled. After the little patient's return to the hospital, I examined the well water microscopically, but failed to find any eggs of the round worm. On Oct. 4th I again saw the child by accident. She was looking well, and had not been troubled with more worms, nor had she again taken to earth-eating. Rugby.

\section{IRRITATION OF THE SKIN FOLLOWING} THE APPLICATION OF VASELINE AS

\section{A SURGICAL DRESSING.}

BY A. W. MAYO ROBSON, F.R.C.S. SURGEON TO THE LEEDS GENERAL INFIRMARY.

ON several occasions within the last two years I have, both in private and in hospital practice, noticed that severe inflammation of the skin has been set up by the application of vaseline as an ointment, which has subsided on applying some other simple dressing. The following examples will serve as illustrations :-

CASE 1.-Mrs. S- attended as an out-patient at the Leeds Infirmary for simple ulcer of the leg, forwhich a Martin's bandage was ordered to be worn during the day, and vaseline to be applied at vight. On her next visit she complained of severe burning in the leg, which was covered with eczema for two inches round the ulcer. The patient was advised to rest and to stop applying the bandage, but to continue the vaseline. Oa her next visit the inflammation had extended over the whole leg. I now thought it might be due to the vaseline, and ordered zinc ointment, which rapidly cured the irritation and enabled the bandage to be worn again, which soon cured the ulcer.

CASE 2.-W. C., aged eight, was brought to my rooms suffering from ophthalmia tarsi, for which I ordered yellow oxide of mercury with vaseline. On the next visit the eye. lids were swollen and odematous, so that the eyeballs could not be seen. I ordered the lids to be washed and simple olive oil to be applied. Two days afterwards, the swelling having subsided, I again ordered yellow oxide of mercury and lard as an ointment, and soon cured the case.

CASE 3-A. Q-, aged four, was brought to my rooms suffering from a wound of the forehead, which was strapped but did not heal by first intention. After a week vaseline was applied; within twelve hours the forehead was covered with small blisters and the skin became brawny. On dis. continuing the ointment and applying some other simple dressing the irritation soon subsided

CASE 4.-A. B- was admitted under my care into the infirmary suffering from scalds of the legs. Vaseline was applied and produced severe irritation, which subsided on its discontinuance.

CASE 5.-C. B—, aged seventeen, was admitted into the intirmary suffering from a smashed leg, for which I performed primary amputation. Near the termination of the case, the granulations being feeble, red lotion was used and vaseline was applied on lint over the lotion to prevent evaporation. It did very well for some time and was healing rapidly until one morning on my ward visit I found the sore inflamed and the adjoining skin cedematous. I could find nothing to account for this, as the same dressing was being used that had been employed for at least a fortnight, until the nurse told me she was using some fresh vaseline, which had a greenish colour but was said to be very pure. On dis. continuing this the inflammation subsided and the sore again began to heal.

I need not enumerate any more cases, but I have no doubt other surgeons will have had the same experience. In the above examples I suppose the irritation may have been due to some impurity in the vaseline, as, if pure, it should be a perfectly bland preparation. But it may be that some skins present the same idiosyncrasy to preparations of vaseline as others do to any application of tar, and this seems to me more likely, since whilst the same vaseline was used in many other cases without producing irritation, in these exceptional casés severe dermatitis was set up.

Leeds.

\section{TUMOURS OF THE TRACHEA.}

BY HENRY CRUTCHLEY, M.D., L.R.C.P. LOND. With Notes BX Dr. Morell Mackenzie.

J. B-, aged forty-nine, married, farm labourer, has had a family of twelve children, ten of whom are now living. His mother died of consumption, and she suffered from scrofulous glands. The patient is a steady, sober man, has enjoyed good health, and has never had syphilis. His present ailment came on gradually about two years ago, when he first perceived a "wheezing and whistling" in his throat upon exertion. His general health continued good, and he followed his employment without inconvenience, except when hurried, till twelve months ago, when he felt that there was "an obstruction in his throat," which kept gradually getting worse, until he had to give up his work a month before last Christmas. He has not had much cough and very little expectoration, but felt roughness and tickling in his throat. $\mathrm{He}$ had no pain or difficulty of swallowing. When I first saw him, though some. what thin, his complexion looked healthy, and there was no lividity. He had an occasional rough, short cough ; no hoarseness. His voice was rather high pitched, and had somewhat of a falsetto character. The larynx did not rise or fall during respiration; there was inspiratory and expiratory stridor, the former scarcely more 